\title{
Quaternaire
}

Revue de l'Association française pour l'étude du

Quaternaire

vol. 28/2 | 2017

Volume 28 Numéro 2

\section{L'œuf ou la poule ? Retour sur le projet Magdatis « Le Magdalénien de la façade atlantique face aux changements environnementaux "}

A chicken-or-egg question. Feedback on the Magdatis project "the Magdalenian from the Atlantic facade facing environmental changes"

Véronique Laroulandie, Sandrine Costamagno, Mathieu Langlais et Jean-Marc Pétillon

\section{OpenEdition}

\section{Journals}

Édition électronique

URL : http://journals.openedition.org/quaternaire/8162

DOI : 10.4000/quaternaire.8162

ISSN : 1965-0795

Éditeur

Association française pour l'étude du quaternaire

Édition imprimée

Date de publication : 29 mai 2017

Pagination : 277-283

ISSN : 1142-2904

Ce document vous est offert par Université Toulouse 2 - Jean Jaurès

Référence électronique

Véronique Laroulandie, Sandrine Costamagno, Mathieu Langlais et Jean-Marc Pétillon, « L'œuf ou la poule ? Retour sur le projet Magdatis « Le Magdalénien de la façade atlantique face aux changements environnementaux » », Quaternaire [En ligne], vol. 28/2 | 2017, mis en ligne le 01 juin 2017, consulté le 09 octobre 2018. URL : http://journals.openedition.org/quaternaire/8162 ; DOI : 10.4000/ quaternaire.8162 


\title{
L'CEUF OU LA POULE? RETOUR SUR LE PROJET MAGDATIS «LE MAGDALÉNIEN DE LA FAÇADE ATLANTIQUE FACE AUX CHANGEMENTS ENVIRONNEMENTAUX »
}

\author{
Véronique LAROULANDIE ${ }^{1}$, Sandrine COSTAMAGNO², Mathieu LANGLAIS ${ }^{1}$ \\ \& Jean-Marc PÉTILLON ${ }^{2}$
}

\begin{abstract}
RÉSUMÉ
L'une des questions qui anime le débat en archéologie paléolithique est de comprendre les relations qui existent entre l'évolution des techniques, des cultures, ou encore des territoires occupés par les groupes humains, et les changements du climat, de la faune et de la flore. Le projet Magdatis avait pour objectif d'apporter des éléments de réponse par l'étude d'un cas privilégié : le Magdalénien moyen et supérieur (vers 19 000-14 000 cal. BP) de l'ouest du Bassin aquitain. Cette région présente de forts contrastes écologiques qui en font un laboratoire idéal pour comparer les comportements des groupes humains dans des milieux différents à la fin des temps glaciaires. Pour cela, un bilan paléoenvironnemental détaillé, des études archéologiques multidisciplinaires et des datations radiocarbone ont été réalisés. Les résultats du projet ont significativement modifié notre vision de l'espace ouest-aquitain au Paléolithique, de ses contraintes environnementales et de son peuplement humain. La Gironde qui se présente alors comme une étendue steppique, semble avoir été désertée pendant deux millénaires, sans doute à cause de dégradations environnementales. Dans les Landes, un désert périglaciaire règne pendant toute la période. Cela explique sans doute la faiblesse de la présence humaine dans cet espace probablement très contraignant pour la vie. En revanche, les zones côtières étaient plus souvent fréquentées et plus intensément exploitées que ce que nous pensions jusqu'ici, attestant de l'existence d'une économie littorale. Dans les basses vallées pyrénéennes, la déglaciation, achevée précocement, libère des paysages ouverts qui sont rapidement occupés par les groupes humains. Ce sont ainsi les variations du milieu qui dessinent la carte des espaces attractifs et répulsifs. En revanche, les industries lithique et osseuse montrent l'existence de réseaux de diffusion dont l'extension n'est pas conditionnée par les obstacles naturels et relève de logiques sociales. La dynamique culturelle observée à cette échelle ne peut pas être reliée simplement aux changements environnementaux.
\end{abstract}

Mots-clés : Préhistoire, culture, chasseurs-collecteurs, Magdalénien, peuplement, climat, environnement, Heinrich 1, datation radiocarbone

\section{ABSTRACT}

A CHICKEN-OR-EGG QUESTION. FEEDBACK ON THE MAGDATIS PROJECT “THE MAGDALENIAN FROM THE ATLANTIC FACADE FACING ENVIRONMENTAL CHANGES"

One of the main issues in Paleolithic archeology is to understand the relationship that the evolution of techniques, cultures, and the territories occupied by human groups, has with changes in the climate, flora, and fauna. The aim of the Magdatis project was to provide elements of answer through the study of a key case: the Middle and Upper Magdalenian (ca. 19.000-14.000 cal. BP) from the Western Aquitaine basin. This region shows sharp ecological contrasts that make it an ideal context to study the behavior of human groups in different environments at the end of the last glaciation. A precise paleoenvironmental survey, multidisciplinary archaeological studies and radiocarbon dating were realized. The results of this project significantly changed our perception of the Western Aquitaine basin in the Paleolithic, of its environmental constraints and human settlements. The Gironde, then mostly a steppe-like region, was apparently deserted for two millennia, probably because of environmental degradation. A periglacial desert laid in the Landes during the whole period considered. This probably explains the small human presence in this region that was a constraining environment for life. Conversely, the coastal areas were more often visited and more intensively exploited than previously thought, attesting the existence of a seashore economy. In the Pyrenean lower valleys, the deglaciation was completed early and opened new landscapes that were rapidly settled by human groups. The environmental changes hence permit to draw a map of attractive and repulsive areas. The lithic and osseous industry, however, show the existence of distribution networks not conditioned by natural obstacles: their extent is instead linked to social dynamics. The cultural dynamics observed on this scale cannot be simply related to environmental changes.

Keywords: Prehistory, culture, climate, hunters-gatherers, Magdalenian, settlement, climate, environment, Heinrich 1, radiocarbon dating

\footnotetext{
${ }^{1}$ PACEA, UMR5199, CNRS et Université de Bordeaux, MCC, FR-33615 PESSAC. Courriels : veronique.laroulandie@u-bordeaux.fr,
} mathieu.langlais@u-bordeaux.fr

${ }^{2}$ TRACES, UMR5608, CNRS et Université Toulouse Jean-Jaurès, Maison de la Recherche, FR-31058 TOULOUSE.

Courriels : costamag@univ-tlse2.fr, petillon@univ-tlse2.fr 


\section{1 - INTRODUCTION}

À travers l'œil de l'archéologue, les archives matérielles issues des fouilles fondent l'histoire des populations humaines anciennes. Peu à peu, les modes de vie, les savoir-faire, les territoires parcourus, les espaces peuplés, l'organisation sociale et les croyances sousjacentes émergent du passé. Cette longue histoire est parsemée de changements qui apparaissent plus ou moins rapides et irréversibles. Au-delà des descriptions factuelles, la recherche s'évertue à qualifier ces changements - rupture, innovation, transition, révolution... sont des concepts fréquemment utilisés - et à en expliquer les mécanismes. Les sciences du paléoenvironnement rejoignent alors des sciences humaines dans des approches interdisciplinaires.

Le projet ANR Magdatis « Des chasseurs-cueilleurs face aux changements environnementaux : le Magdalénien de la façade atlantique au Tardiglaciaire " s'inscrit parmi ces tentatives pour la période préhistorique. Outre le fait que la thématique générale « Homme/Environnement » est fortement inspirée, si ce n'est orientée, par les préoccupations actuelles de notre société, plusieurs critères ont présidé au choix particulier du sujet.

Pour la période considérée, 19 000-14 $000 \mathrm{cal}$. BP, la calibration des dates radiocarbone présente une assez bonne résolution (Reimer et al., 2013), permettant d'envisager la mise en parallèle, a priori selon une même chronologie, des données culturelles, environnementales et climatiques pouvant être datées par différentes méthodes.
Ces archives sont marquées par des changements, autorisant l'observation de synchronies ou d'asynchronies, voire la déduction de l'existence ou non de liens de causalité. Du point de vue climatique, d'après les évènements climatiques enregistrés dans les carottes glaciaire du Groenland et la chronologie GICC05 proposée par Rasmussen et al. (2014), la seconde partie du Greenland Stadial 2.1 (GS-2.1b) est suivie vers $17480 \pm 330$ b2k du GS-2.1a, puis à partir de $14692 \pm 186$ b2k du réchauffement du GI-1e. Cette période comprend également la débâcle glaciaire du stade d'Heinrich 1 (HS1), événement complexe et polyphasé, enregistré dans les carottes marines (Stanford et al. 2011 ; Naughton et al., 2016). Les données continentales montrent une recomposition des biocénoses végétales avec le passage, vers $14700 \mathrm{cal}$. BP, de la palynozone du Dryas ancien - conséquence continentale de HS1 - au Bølling. Le monde animal évolue en conséquence, selon un rythme qui reste à préciser, avec le remplacement des faunes de milieu ouvert et froid par des faunes à caractère plus tempéré. Du point de vue culturel, l'Europe de l'ouest voit se développer l'un des plus emblématiques courants du Paléolithique supérieur, le Magdalénien, dans ses phases moyenne et supérieure. Il est marqué notamment par une remarquable technologie sur grandes lames en silex, une industrie osseuse abondante et variée, un art mobilier et pariétal exceptionnel...

La région d'étude, l'ouest du Bassin aquitain, montre des milieux contrastés, plaine et colline au nord et à l'est, piémont pyrénéen au sud, désert sableux au centre et littoral atlantique à l'ouest (fig. 1). Cette diversité

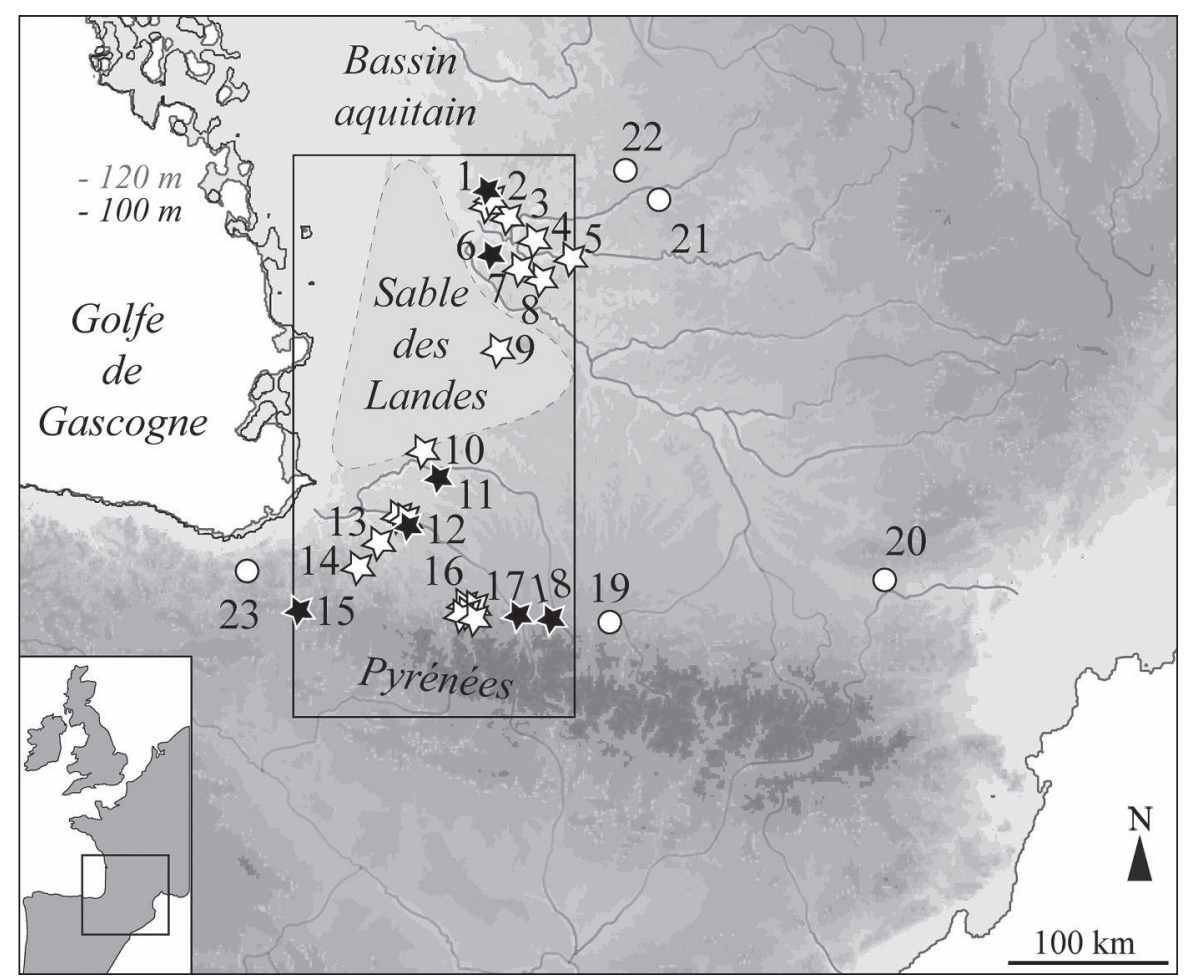

Fig. 1 : Distribution des sites magdaléniens dans l'aire géographique considérée.

Étoiles blanches : sites étudiés; étoiles noires : sites non étudiés ; cercles blancs : sites étudiés hors de l'aire géographique centrale du projet. 1/ La Lustre ; 2/ Roc-de-Marcamps ; 3/ Les Fées, Saint-Germain-la-Rivière ; 4/ Fongaban ; 5/ Le Morin ; 6/ : Jaurias ; 7/ Moulin-Neuf ; 8/ Faustin, Fontarnaud ; 9/La Honteyre ; 10/ Tizon ; 11// Brassempouy ; 12/ Sordes sites : Dufaure, Duruthy, Grand Pastou ; 13/ Bourrouilla ; 14/ Isturitz ; 15/ Abauntz ; 16/ sites du bassin d'Arudy : Bignalats, Espalungue, Laa 2, Poeymaü, Saint-Michel, Sainte-Colome ; 17/ Espélugues ; 18/ Aurensan ; 19/ Troubat ; 20/ Gazel ; 21/ Chancelade ; 22/ Pont d'Ambon ; 23/ Santa Catalina (en gras les sites clés).

Fig. 1: Distribution of the Magdalenian sites in the area investigated. White stars: studied sites; black stars: unstudied sites; white circles: sites studied outside the core area of the project (key sites in bold). 
permet de documenter les comportements des groupes humains dans différents biotopes. Cette région présente également l'avantage de livrer un riche corpus de sites et de données, hérité des nombreux travaux antérieurs. Il s'agit donc d'un cas d'étude privilégié, et, qui plus est, de l'une des plus anciennes périodes de la Préhistoire où une analyse systémique du rôle des changements environnementaux sur l'évolution des territoires et de l'économie des groupes humains peut-être envisagée.

\section{2 - MOYENS, MATÉRIEL ET MÉTHODES}

Le projet ANR Magdatis a duré officiellement 44 mois et a réuni 36 personnes (voir remerciements).

Des bilans paléoenvironnementaux ont été dressés afin de mettre à disposition des préhistoriens des données récentes et critiquées. L'accent a été mis sur l'extension et la formation du Sable des Landes, sur la question de l'existence régionale d'un pergélisol, sur la chronologie de la déglaciation des vallées du versant nord des Pyrénées et sur l'évolution des biocénoses végétales et animales.

Un fort investissement a été consacré au renouvellement des données archéologiques primaires, d'une part en réexaminant des collections issues d'anciennes fouilles et, d'autre part, en intégrant des nouvelles données de terrain (fig. 1). Ces analyses chronophages, basées sur des sites-clés, se sont focalisées sur la caractérisation de l'évolution des choix techno-économiques. Afin de contrôler la fiabilité et la représentativité du matériel analysé, nous avons adopté une approche archéostratigraphique de la distribution des vestiges et avons effectué des études taphonomiques des ensembles osseux. Nous avons également conduit des études technologiques, typologiques et économiques des équipements en pierre et en matières osseuses, ainsi que des analyses archéozoologiques.

Parallèlement, un corpus de 69 dates radiocarbone par AMS provenant de 18 sites a été constitué à partir d'échantillons scrupuleusement sélectionnés : ossements d'espèces déterminées, éléments particuliers d'industrie osseuse et restes humains. Ces dates ont permis, respectivement, de préciser la chronologie de la recomposition des biocénoses animales dans la région, de caler l'évolution de l'outillage osseux et de dater certaines pratiques mortuaires (Barshay-Szmidt et al., 2016 ; Costamagno et al., 2016 ; Pétillon, 2016a). Ces dates ont été calibrées à $2 \sigma$ à l'aide du logiciel OxCal 4.2.4 (Bronk Ramsey, 2009) et de la courbe IntCal13 (Reimer et al., 2013); elles sont exprimées en cal. BP pour faciliter les comparaisons avec les chronologies environnementales données en âges calendaires. Les limites des phases culturelles mises en évidence ont été précisées en utilisant le modèle bayésien "Contiguous Trapezium » d'Oxcal 4.2.4 qui apparaissait bien adapté aux données traitées (BarshaySzmidt et al., 2016). Ce modèle permet de proposer une succession chronologique statistiquement fiable des phases sans exclure leur possible recouvrement (Lee \& Bronk Ramsey, 2012). Une période de flou résultant du type de sites analysés (palimpsestes), des méthodes de datation et de modélisation apparaît donc entre chaque phase.

Enfin, un effort de croisement de ces différentes données a été fait en restant conscient qu'il s'agit d'une étape de la recherche fortement soumise aux incertitudes inhérentes aux méthodes de datation et aux modélisations chronologiques choisies.

\section{3 - RÉSULTATS}

Étant donné que plus d'une trentaine d'articles exposent d'ores et déjà les conclusions du projet Magdatis [liste dans Pétillon et al., 2016a ; mise à jour sur : https :// univ-tlse2.academia.edu/Magdatisproject], nous focaliserons notre attention sur quelques résultats impliquant le croisement des données environnementales et culturelles, et les développements envisageables.

\section{1 - UNE ÉVOLUTION À PLUSIEURS VITESSES}

Le registre des activités liées à la chasse illustre différentes vitesses dans la dynamique évolutive du Magdalénien. Tandis que certains comportements apparaissent globalement stables, et donc indépendants des variations climatiques et des réponses environnementales enregistrées, d'autres se modifient au cours des 5000 ans observés. Par exemple, pendant toute la période considérée, l'utilisation du propulseur et de projectiles constitués de pointes osseuses armées latéralement de microlithes se présente comme la norme pour la chasse des ongulés (Langlais et al., 2016 ; Pétillon, 2016a). Des variations discrètes de formes et de décors des objets s'observent, mais elles relèveraient plus de phénomènes de mode - avec les implications sociales que ces derniers supposent - que d'une révolution technique ou économique. Autant que l'on puisse en juger, le traitement du grand gibier semble également relativement stable avec une exploitation poussée des carcasses tout au long de la période considérée (e.g. Kuntz et al., 2016).

Parallèlement, des évolutions typologiques et technologiques apparaissent clairement dans le registre de l'industrie lithique et osseuse et sous-tendent un découpage en quatre phases nommées Magdalénien moyen ancien (MMA), moyen récent (MMR), supérieur ancien (MSA) et supérieur récent (MSR) ; (voir détails dans Langlais et al., 2016 ; Pétillon, 2016a). La chronologie de ces phases a été précisée grâce aux datations radiocarbone (fig. 2). Des arythmies s'observent selon le registre pris en considération. À un certain niveau, ces phases peuvent visuellement apparaître comme contemporaines d'épisodes climatiques, notamment l'HS1, mais le flou généré par les incertitudes des méthodes de datation ne permet guère de dépasser cette impression.

Enfin, plusieurs innovations dans les armes de chasse voient le jour durant le Magdalénien supérieur et perdureront, avec des changements de forme, dans l'Azilien. Il s'agit notamment des pointes lithiques réalisées sur petites lames et des pointes osseuses barbelées qui sont 


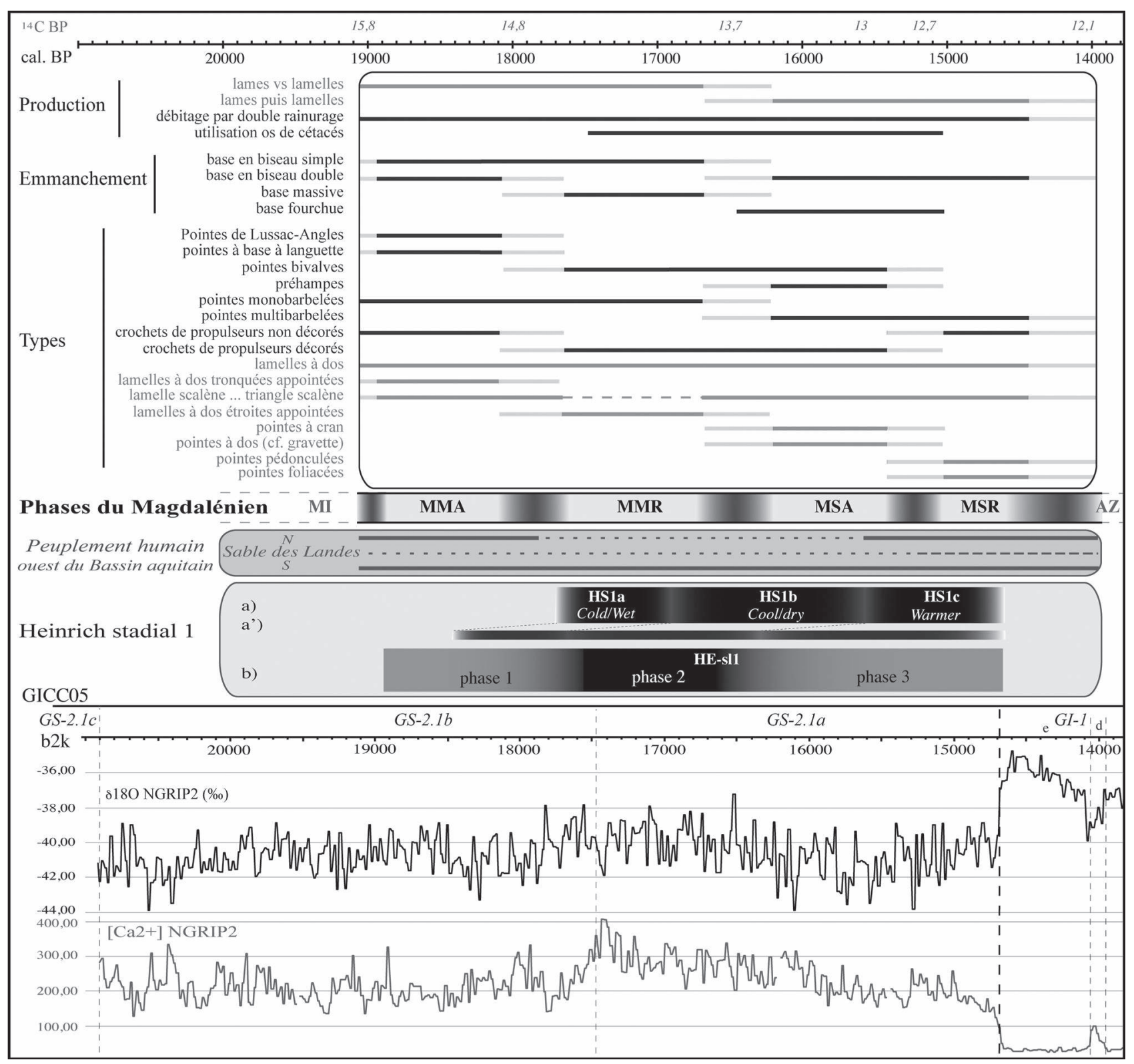

Fig. 2 : Changements culturels et climatiques entre 19000 et 14000 cal. BP dans le sud-ouest de la France.

Données sur l'équipement de chasse osseux (gris foncé) et lithique (gris clair) d'après Pétillon (2016) et Langlais et al. (2016) ; MI : Magdalénien inférieur; MMA : Magdalénien moyen ancien; MMR : Magdalénien moyen récent ; MSA : Magdalénien supérieur ancien ; MSR : Magdalénien supérieur récent. Chronologie des phases culturelles et occupation humaine d'après Barshay-Szmidt et al. (2016); les zones floues indiquent les incertitudes chronologiques. Peuplement du Bassin aquitain ; continu (trait plein); discontinu (trait pointillé). Chronologie du Stade d'Heinrich 1 ; a/ d'après Naughton et al. (2016) avec correction adaptée de l'âge réservoir, a'/ avec correction standard de l'âge réservoir et b/ Stanford et al. (2011). Evénements climatiques dans les carottes glaciaires du Groenland : GS-2 (Stade 2) ; GI-1 (interstade 1) ; b2k (avant 2 000) ; chronologie GICC05 d'après Rasmussen et al. (2014); données $\delta^{18} \mathrm{O}$ et $[\mathrm{Ca} 2+]$ d'après Rasmussen et al. (2014) et Seierstad et al. (2014).

Fig. 2: Cultural and climatic changes between 19.000 and 14.000 cal. BP in southwest France. Osseous (dark grey) and lithic (light grey) hunting implements data from Pétillon (2016) and Langlais et al. (2016); MI: Late Magdalenian; MMA: Early Middle Magdalenian; MMR: Late Middle Magdalenian; MSA: Early Upper Magdalenian; MSR: Late Middle Magdalenian. Cultural phases and human occupation data from Barshay-Szmidt et al. (2016); Blurred areas indicate the chronological uncertainties. Aquitain Bassin Settlement; continuous (solid line); discontinuous (dotted line). Heinrich stadial 1 chronology from a/ Naughton et al. (2016) with adapted correction of the reservoir age, a $/$ with standard reservoir age, and b/ Stanford et al. (2011). Climatic events from Greenland ice core: GS-2 (Greenland stadial 2); GI-1 (Greenland interstadial 1); b2k (before 2000 years); GICC05 chronology from Rasmussen et al. (2014); $\delta^{18} \mathrm{O}$ and $[\mathrm{Ca}+]$ data from references in Rasmussen et al. (2014) and Seierstad et al. (2014).

utilisées conjointement aux armatures composites. Cette phase voit également la domestication du loup (BoudadiMaligne et al., 2012), processus changeant probablement les relations - et les représentations - que les hommes entretiennent avec le monde animal. Une intensification de la consommation des petits gibiers, dont les racines sont néanmoins plus anciennes, est aussi perceptible dans l'espace considéré (e.g. Costamagno et al., 2008 ; Langlais et al., 2012 ; Laroulandie, 2016). Bien que cela reste à démontrer, il est envisageable que le développement d'espèces végétales héliophiles et pionnières à la fin du Dryas ancien et au Bølling (e.g. Naughton et al., 2016) ait constitué un facteur favorisant la démographie des petits animaux s'en nourrissant, tels les lagopèdes ou le lièvre variable. Le cas échéant, cette disponibilité accrue aurait rendu possible l'intensification de leur exploitation. Mais il est difficile de conclure qu'elle a contraint les hommes. Les ongulés restent en effet la source carnée 
principale et l'utilisation des petits animaux au-delà du domaine strict de l'alimentation interroge sur les motivations de leur acquisition. En d'autres termes, le cocktail des causes impliquées dans leur exploitation reste encore à préciser dans sa complexité. Se procurer ces petits animaux a nécessité de nouvelles stratégies et la question du chien comme auxiliaire de chasse, ou de l'utilisation de nouvelles armes, se pose. Là encore, les conclusions ne doivent pas être trop hâtives et globales ; il serait réducteur de considérer qu'un comportement unique puisse caractériser la diversité des petits animaux acquis.

\section{2 - UN TERRITOIRE EN MOSAÏQUE AU PEU- PLEMENT HÉTÉROGÈNE}

Dans la région considérée, la remontée du niveau marin, de près de 120 mètres entre 20000 cal. BP et l'actuel, a entraîné un fort recul de la frange littorale atlantique (fig. 1). Les activités qui ont pu s'y dérouler pourraient, de fait, apparaître complètement inaccessibles aux préhistoriens. Néanmoins des représentations et des indices matériels (par exemple os de cétacé, coquillages) qui ont circulé vers l'intérieur des terres constituent une preuve de la fréquentation de cet espace et suggèrent l'existence d'une économie littorale durant le Magdalénien (voir bilan dans Pétillon, 2016b). Il est impossible de savoir si les chasseurs-collecteurs ont perçu, grâce à la transmission des connaissances d'une génération à l'autre, la montée du niveau marin. De l'ordre de $20 \mathrm{~m}$ sur les 5000 ans considérés, l'élévation du niveau marin a atteint, il y a 16000 ans, $15 \mathrm{~mm}$ par an, soit 5 fois plus qu'aujourd'hui (Lambeck et al., 2002). Quoi qu'il en soit, l'espace littoral a évolué et ces populations ont dû s'y adapter.

Durant cette période, la région du Sable des Landes fonctionnait comme un désert périglaciaire avec une couverture sableuse entourée de formations loessiques. De forts vents soufflant principalement depuis le nordouest alimentaient ce système (Bertran et al., 2013 ; Sitzia et al., 2015). Cette région a constitué une zone répulsive limitant le territoire occupé par les groupes humains durant tout le Paléolithique supérieur (Bertran et al., 2013). Le gisement de la Honteyre, fouillé par M. Lenoir, indique cependant que la frange nord de cette formation sableuse a été fréquentée par les nomades durant le MSR (Belbéoc'h \& Lenoir, 2011). Les silex principalement exploités (grain de mil) ont été transportés sous la forme de rognons sur plus de $100 \mathrm{~km}$ en provenance du Nord. Très peu de matériaux locaux sont représentés, ce qui pourrait indiquer un campement de courte durée mais dont l'outillage témoigne d'un habitat de type résidentiel (Langlais et al., 2016; Gourc et al., 2016).

Concernant le piémont ouest-pyrénéen et en particulier le bassin d'Arudy, le peuplement magdalénien débute vers 20000 cal. BP, soit plus anciennement qu'il n'était précédemment envisagé (Pétillon et al., 2015). Il suivrait donc de peu la déglaciation de la vallée et l'ouverture de nouveaux espaces.

$\mathrm{Si}$ les dates radiocarbone montrent un peuplement relativement continu du piémont ouest-pyrénéen, il n'en est pas de même au nord du Sable des Landes, dans l'actuelle Gironde (Barshay-Szmidt et al., 2016). Dans cette zone, un hiatus de près de 2000 ans, situé grosso modo entre 17500 et 15500 cal. BP, apparait clairement (fig. 2). Pendant ce temps, se développe au sud du Sable le MMR dont les marqueurs, pourtant recherchés, sont absents au nord. Ce hiatus est contemporain de la première moitié de la péjoration climatique du Dryas ancien. Selon la chronologie marine (fig. 2), il correspond également à la phase 2 du stade d'Heinrich 1 (Stanford et al. 2010), au HS1b (Naughton et al., 2016) - tenant compte de la correction standard de l'effet réservoir -, ou encore au HS1a (Naughton et al., 2016) basé cette fois sur une valeur corrigée de l'effet réservoir. Des signatures sédimentaires périglaciaires (cryoturbation et réseaux de petits polygones de cryodessiccation) observées en grotte et en plein air dans le nord du Bassin aquitain marquent également le refroidissement (Lenoble et al., 2012), sans toutefois indiquer le développement d'un véritable pergélisol (Andrieux et al., 2016). Par ailleurs, de part et d'autre de ce hiatus, les archéofaunes évoluent considérablement, indiquant un changement environnemental : l'antilope saïga et les bovinés, qui occupaient le haut des tableaux de chasse durant le MMA, laissent la place au cheval et au renne durant le MSA et le MSR (e.g. Delpech, 1983 ; Kuntz et al., 2016). Il est donc probable que le hiatus chronologique observé marque un changement dans le peuplement de cette région, devenue inhospitalière, en tout cas pour des occupations suffisamment longues et fréquentes pour être détectées par les préhistoriens (Pétillon et al., 2016a). Seule la présence de quelques pièces en silex charentais (i.e. grain de mil) trouvées au sud pourrait indiquer que cet espace a été traversé. En revanche, au MMR, le maintien d'importants axes de circulation des silex entre la région du Bergeracois et les piémonts au sud témoigne d'une perduration de certains réseaux identifiés au MMA (Langlais et al., 2016). Ces axes de diffusion des matériaux permettent de soutenir l'exigence culturelle du système de production de grandes lames normées dans une région où de tels volumes en silex font défaut. Ceci implique l'existence de déplacements des groupes sur d'importantes distances ou d'échanges supportés par des réseaux sociaux. Ces déplacements semblent s'affranchir des limites des écosystèmes et reflètent davantage les choix économiques et les logiques sociales qui les portent. La valeur accordée aux grandes lames se manifeste notamment par l'existence de caches trouvées dans des contextes particuliers de grottes ornées et dont l'utilisation dépasse la sphère domestique (voir bilan dans Angevin \& Langlais 2009).

\section{4 - EN GUISE DE CONCLUSION}

Dans l'espace-temps retenu, les quelques exemples développés ici mettent en lumière l'influence des conditions environnementales sur l'évolution du peuplement humain. Les causes des changements techniques apparaissent, en revanche, plus difficiles à appréhender car 
ces derniers résultent, en grande partie, d'une dynamique propre mettant en jeu un bagage de normes culturelles acquises depuis des générations.

À l'échelle relativement courte des phénomènes humains considérés ici, on peut se questionner sur le poids des incertitudes chronologiques de chaque registre d'archive dans les interprétations. En d'autres termes, pour explorer la complexité de la relation homme/environnement à l'échelle des changements socio-économiques mis en évidence, les chronologies glaciaire, marine et continentale sont-elles réellement comparables ? Clairement, les efforts doivent se poursuivre pour affiner de manière indépendante la chronologie des phénomènes observés et préciser les méthodes de synchronisation utilisées (e.g. Blaauw, 2012), ce qui est résolument une affaire interdisciplinaire. La poursuite de l'acquisition de proxies environnementaux locaux à l'échelle des sites (géoarchéologie, spéléothèmes, associations de micromammifères ou de malacofaune, analyses isotopiques...) et à l'échelle régionale est nécessaire. Elle devrait permettre, dans la limite des méthodes employées, d'évaluer plus précisément la nature et l'inertie des réponses des écosystèmes comme les degrés de résilience des sociétés humaines face aux changements climatiques globaux. Cette quête nécessite parallèlement de considérer, autant que possible, l'impact des paradigmes sous-jacents à nos travaux.

\section{REMERCIEMENTS}

Le projet Magdatis a bénéficié du financement de l'Agence Nationale de la Recherche (ANR 2011 BSH3 0005). En plus des auteurs, ce projet a réuni les membres C. Barshay-Szmidt, P. Bertran, C. Birouste, M. Boudadi-Maligne, S. Caux, F.-X. Chauvière, D. Cochard, M. Dachary, M. Delmas, E. Discamps, I. Elorrieta, L. Feyfant, D. Galop, L. Gourc, D. HenryGambier, D. Kuntz, F. Lacrampe-Cuyaubère, A. Lefebvre, M. Lenoir, J.-B. Mallye, G. Marsan, F. Martin, H. Martin, C. Masset, F. Naughton, C. Normand, E. Pubert, W. Rendu, A. Royer, A. Sécher, L. Sitzia et A. Tarriño qui sont chaleureusement remerciés. Cet article a bénéficié des conseils précis et avisés de $\mathrm{W}$. Banks ainsi que des corrections de A. Landais.

\section{RÉFÉRENCES BIBLIOGRAPHIQUES}

ANDRIEUX E., BERTRAN P. \& SAITO K., 2016 - Spatial analysis of the French Pleistocene permafrost by a GIS database. Permafrost and periglacial processes, 27 (1), 17-30.

ANGEVIN R. \& LANGLAIS M., 2009 - Où sont les lames ? Enquête sur les " caches " et "dépôts " de lames du Magdalénien moyen (15 000-13 500 BP). In S. Bonnardin, C. Hamon, M. Lauwers \& B. Quilliec (dir.), Du matériel au spirituel : réalités archéologiques et historiques des "dépôts " de la Préhistoire à nos jours. Actes des $\mathrm{XXIX}^{\mathrm{e}}$ Rencontres internationales d'Archéologie et d'Histoire d'Antibes, octobre 2008, APDCA, Antibes, 223-242.

BARSHAY-SZMIDT C., COSTAMAGNO S., HENRY-GAMBIER D., LAROULANDIE V., PÉTILLON J.-M., BOUDADIMALIGNE M., KUNTZ D., LANGLAIS M. \& MALLYE J.-B., 2016 - New extensive focused AMS 14C dating of the Middle and
Upper Magdalenian of the western Aquitaine/Pyrenean region of France (ca. 19-14 ka cal BP): Proposing a new model for its chronological phases and for the timing of occupation. Quaternary International 414, 62-91.

BELBÉOC'H G. \& LENOIR M., 2011 - Le Paléolithique supérieur et l'Epipaléolithique dans la partie nord des Landes de Gascogne (secteur d'Hostens). In J.-C. Merlet \& J.-P. Bost (dir.), De la Lagune à l'Airial : Le Peuplement de la Grande Lande. Editions de la Fédération Aquitania, Bordeaux, 61-76.

BERTRAN P., SITZIA L., BANKS W.E., BATEMAN M.D., DEMARS P.-Y., HERNANDEZ M., LENOIR M., MERCIER N. \& PRODEO F., 2013 - The Landes de Gascogne (southwest France): periglacial desert and cultural frontier during the Palaeolithic. Journal of Archaeological Science, 40 (5), 2274-2285.

BLAAUW M., 2012 - Out of tune: the dangers of aligning proxy archives. Quaternary Science Reviews, 36, 38-49.

BOUDADI-MALIGNE M., MALLYE J.-B., LANGLAIS M. \& BARSHAY-SZMIDT C., 2012 - Des restes de chien magdaléniens à l'abri du Morin (Gironde, France) : implications socio-économiques d'une innovation zootechnique. Paléo, 23, 39-54.

BRONK RAMSEY C., 2009 - Bayesian analysis of radiocarbon dates. Radiocarbon, 51 (1), 337-360.

COSTAMAGNO S., COCHARD D., FERRIÉ J.-G., LAROULANDIE V., BARBAZA M., CAZALS N., LANGLAIS M., VALDEYRON N., DACHARY M., GALOP D., MARTIN H. \& PHILIBERT S., 2008 - Nouveaux milieux, nouveaux gibiers, nouveaux chasseurs ? Evolution des pratiques cynégétiques dans les Pyrénées du Tardiglaciaire au début du Postglaciaire. Bulletin de la Société Préhistorique Française, 105 (1), 17-27.

COSTAMAGNO S., BARSHAY-SZMIDT C., KUNTZ K., LAROULANDIE V., PÉTILLON J.-M., BOUDADI-MALIGNE M., LANGLAIS M., MALLYE J.-B. \& CHEVALLIER A., 2016 - Reexamining the timing of reindeer disappearance in southwestern France in the larger context of late glacial faunal turnover. Quaternary International, 414, 34-61.

DELPECH F., 1983 - La faune du Paléolithique supérieur dans le SudOuest de la France. Cahier du Quaternaire 6, CNRS, Centre régional de publication de Bordeaux, $453 \mathrm{p}$.

GOURC L., LANGLAIS M., SITZIA L., CAUX S., BELBEOC'H G. \& LENOIR M., 2016 - La Honteyre (Le Tuzan, Gironde) : une occupation de chasseurs-collecteurs magdaléniens en contexte de désert sableux, Bulletin de la Société Préhistorique Française, 113 (4), 713-735.

KUNTZ D., COSTAMAGNO S., FEYFANT L., MARTIN F., 2016 - The exploitation of ungulates in the Magdalenian in the Entre-Deux-Mers (Gironde, France). Quaternary International, 414, $108-158$.

LAMBECK K., YOKOYAMA Y. \& PURCELL T., 2002 - Into and out of the Last Galcial Maximum: sea-level change during Oxygen Isotopes Stages 3 and 2. Quaternary Science Reviews, 21 (1-3), 343-360.

LANGLAIS M., COSTAMAGNO S., LAROULANDIE V., PÉTILLON J.-M., DISCAMPS E., MALLYE J.-B., COCHARD D. \& KUNTZ D., 2012 - The evolution of Magdalenian societies in South-West France between 18,000 and 14,000 cal BP: changing environments, changing tool kits. Quaternary International, 272-273, 138-149.

LANGLAIS M., SÉCHERA., CAUX S., DELVIGNE V., GOURC L., NORMAND C. \& SÁNCHEZ DE LA TORRE M., 2016 - Lithic tool kits: A Metronome of the evolution of the Magdalenian in southwest France (19,000-14,000 cal BP). Quaternary International, 414, 92-107.

LAROULANDIE V., 2016. Hunting fast-moving, low-turnover small game: The status of the snowy owl (Bubo scandiacus) in the Magdalenian. Quaternary International, 414, 174-197.

LEE S. \& BRONK RAMSEY C. 2012 - Development and Application of the Trapezoidal Model for Archaeological Chronologies. Radiocarbon, 54 (1), 107-122.

LENOBLE A., BERTRAN P., MERCIER N. \& SITZIA L., 2012 - Le site du Lac Bleu et la question de l'extension du pergélisol en France au Pléistocène supérieur. In P. Bertran \& A. Lenoble (dir.), Quaternaire continental d'Aquitaine: un point sur les travaux récents. Guide de l'Excursion AFEQ-ASF 2012, 109-121.

NAUGHTON F., SANCHEZ GOÑI M.F., RODRIGUES T., SALGUEIRO E., COSTAS, S., DESPRAT S., DUPRAT J., MICHEL E., ROSSIGNOL L., ZARAGOSI S., VOELKER A.H.L. \& ABRANTES F., 2016 - Climate variability across the last deglaciation in NW Iberia and its margin. Quaternary International, 414, 9-22.

PÉTILLON J.-M. 2016a - Technological evolution of hunting implements among Pleistocene hunter-gatherers: Osseous projectile points 
in the middle and upper Magdalenian (19-14 ka cal BP). Quaternary International, 414, 108-134.

PETILLON J.-M. 2016b - Life on the shore of the Bay of Biscay in the Late Upper Paleolithic: towards a new paradigm. In C. Dupont \& G. Marchand (dir.), Archéologie des Chasseurs-cueilleurs Maritimes. Paris, Société préhistorique française, 23-36.

PÉTILLON J.-M., LANGLAIS M., KUNTZ D., NORMAND C., BARSHAY-SZMIDT C., COSTAMAGNO S., DELMAS M., LAROULANDIE V. \& MARSAN G., 2015 - The human occupation of the northwestern Pyrenees in the Late Glacial: new data from the Arudy basin, lower Ossau valley. Quaternary International, 364, 126-143.

PÉTILLON J.-M., LAROULANDIE V., COSTAMAGNO S. \& LANGLAIS M. 2016 - Testing environmental determinants in the cultural evolution of hunter-gatherers: a three-year multidisciplinary project on the occupation of the western Aquitaine basin during the Middle and Upper Magdalenian (19-14 kyr cal BP). Quaternary International, 414, 1-8.

RASMUSSEN S.O., BIGLER M., BLOCKLEY S.P., BLUNIER T., BUCHARDT S.L., CLAUSEN H.B., CVIJANOVIC I., DAHL-JENSEN D., JOHNSEN S.J., FISCHER H., GKINIS V., GUILLEVIC M., HOEK W.Z., LOWE J.J., PEDRO J.B., POPP T., SEIERSTAD I.K., STEFFENSEN J.P., SVENSSON A.M., VALLELONGA P., VINTHER B.M., WALKER M.J., WHEATLEY J.J. \& WINSTRUP M., 2014 - A stratigraphic framework for abrupt climatic changes during the Last Glacial period based on three synchronized Greenland ice-core records: refining and extending the INTIMATE event stratigraphy. Quaternary Science Reviews, 106, 14-28.
REIMER P. J., BARD E., BAYLISS A., BECK J.W., BLACKWELL P. G., BRONK RAMSEY C., BUCK C. E., CHENG H., EDWARDS R. L., FRIEDRICH M., GROOTES P. M., GUILDERSON T. P., HAFLIDASON H., HAJDAS I., HATTE C. HEATON T. J., HOFFMANN D. L., HOGG A. G., HUGHEN K. A., KAISER K. F., KROMER B., MANNING S. W., NIU M. REIMER R. W., RICHARDS D. A., SCOTT E. M., SOUTHON J. R., STAFF R. A., TURNEY C. S. M. \& VAN DER PLICHT J., 2013 - Intcal13 and Marine13 radiocarbon age calibration curves 0-50,000 years Cal BP. Radiocarbon, 55 (4), 1869-1887

SEIERSTAD I.K., ABBOTT P.M., BIGLER M., BLUNIER T., BOURNE A.J., BROOK E., BUCHARDT S.L., BUIZERT C., CLAUSEN H.B., COOK E., DAHL-JENSEN D., DAVIES S.M., GUILLEVIC M., JOHNSEN S.J., PEDERSEN D.S., POPP T.J, RASMUSSEN S.O., SEVERINGHAUS J.P., SVENSSON A. \& VINTHER B.M., 2014 - Consistently dated records from the Greenland GRIP, GISP2 and NGRIP ice cores for the past 104 ka reveal regional millennial-scale $\delta^{18} \mathrm{O}$ gradients with possible Heinrich event imprint. Quaternary Science Reviews, 106, 29-46.

SITZIA L., BERTRAN P., BAHAIN J.-J., BATEMAN M.D., HERNANDEZ M., GARON H., DE LAFONTAINE G. MERCIER N., LEROYER C., QUEFFELEC A. \& VOINCHET P., 2015 - The quaternary coversands of southwest France. Quaternary Science Reviews, 124, 84-105.

STANFORD J.D., ROHLING E.J., BACON S., ROBERTS A.P., GROUSSET F.E. \& BOLSHAW M. 2011 - A new concept for the paleoceanographic evolution of Heinrich event 1 in the North Atlantic. Quaternary Science Reviews, 30, 1047-1066. 